\title{
Redox-active Monolayers Deposited on Gold Electrode Surface-Universal Platforms for Electrochemical Sensing
}

\author{
Jerzy Radecki and Hanna Radecka* \\ Institute of Animal Reproduction and Food Research, Polish Academy of Sciences, \\ Tuwima 10, 10-748 Olsztyn, Poland
}

(Received October 24, 2019; accepted February 28, 2020)

Keywords: functionalization of gold electrode surface, redox-active complexes, interfacial recognition, electrochemical signal generation

Over the last decade, the intermolecular recognition of anions in water has been attracting the attention of numerous scientific groups involved in supramolecular chemistry. Most of the literature is on the recognition of anions in one organic phase. The development of systems for the recognition of anions in aqueous media is still a challenging task. In this review, examples of sensors and biosensors based on redox-active monolayers destined for the determination of anions, oligonucleotide sequences and antibodies, as well as kinase inhibitors, in aqueous media will be presented. The very high sensitivity in the picomolar range will be supported by very high values of the interphase receptor-target compound binding constant and reaction coupling efficiency. The mechanism of communication between the redox center and the receptor-target analyte complex, as well as analytical signal generation, will also be discussed.

\section{Introduction}

The extraordinary selectivity and sensitivity of molecular recognition processes occurring in biological systems are observed in water, and this remains an inexhaustible source of inspiration for researchers dealing with supramolecular and analytical chemistry. ${ }^{(1)}$ Generally, intermolecular recognition processes rely on the formation of hydrogen bonds. Also, interactions such as electrostatic, Van der Waals, Lewis acid-base and pi-pi interactions play a crucial role.

Water molecules can act as both hydrogen bond donors and acceptors. Therefore, soluble polar molecules are highly hydrated. This feature provides a major challenge in the development of functional systems in aqueous media, and consequently, it is important to investigate how the involvement of water molecules in recognition processes can be minimized in certain cases and exploited in others. Thus, there is a great need for the development of new, fast, cheap "smart systems" that can monitor biologically relevant compounds in water and might be used not only in research laboratories as a tool for the observation of recognition processes at the interface, but also in future applications for the formation of electrochemical

*Corresponding author: e-mail: h.radecka@pan.olsztyn.pl

https://doi.org/10.18494/SAM.2020.2662 
sensors and biosensors destined for medical diagnosis, food quality control, and environmental screening.

In the context of this goal, the preparation of well-defined functional surfaces of solid supports is one of the most crucial tasks. The new materials with chemical as well as physical properties precisely designed for special purposes are fundamental for developing a new sensor technology. Electrochemical sensors are very promising, because they allow for the direct conversion of a supramolecular recognition process into an electronic signal. In addition, they possess essential advantages, such as excellent detection limits, small sample volume consumption, ability to be used in turbid biofluids, suitability for rapid measurement, and ease of miniaturization. ${ }^{(2)}$

The analytically active layer consisting of molecules that enable the recognition of a particular target or group of targets determines the selectivity of electrochemical sensors. The transducer that converts an energy signal resulting from an intermolecular recognition into a form of energy that is readable by the readout device determines the sensor sensitivity (Fig. 1).

This review article will be focused on sensors and biosensors created using the redox-active monolayers deposited on gold electrodes. One of their main advantages is the elimination of the necessity of using redox markers in a sample solution. The faradaic current originating from redox reactions of redox centers is essentially unaffected by monolayer defects. The covalent immobilization of the redox centers on the electrode surface eliminates the effect of diffusion on the cyclic voltametry response. As a consequence, the double-layer correction for the surface concentration versus the bulk concentration is unnesesery. ${ }^{(3,4)}$

The spontaneous creation of covalent bounds between the sufur atoms present in various thio compounds and nobel metals, such as gold, silver, platinum, and mercury, discovered by Allara and co-workers, ${ }^{(5,6)}$ has given rise to the the possiblity of applying surface functionalization to achieve their desired chemical and electrochemical properties. The advantage of $\mathrm{Au}-\mathrm{S}$
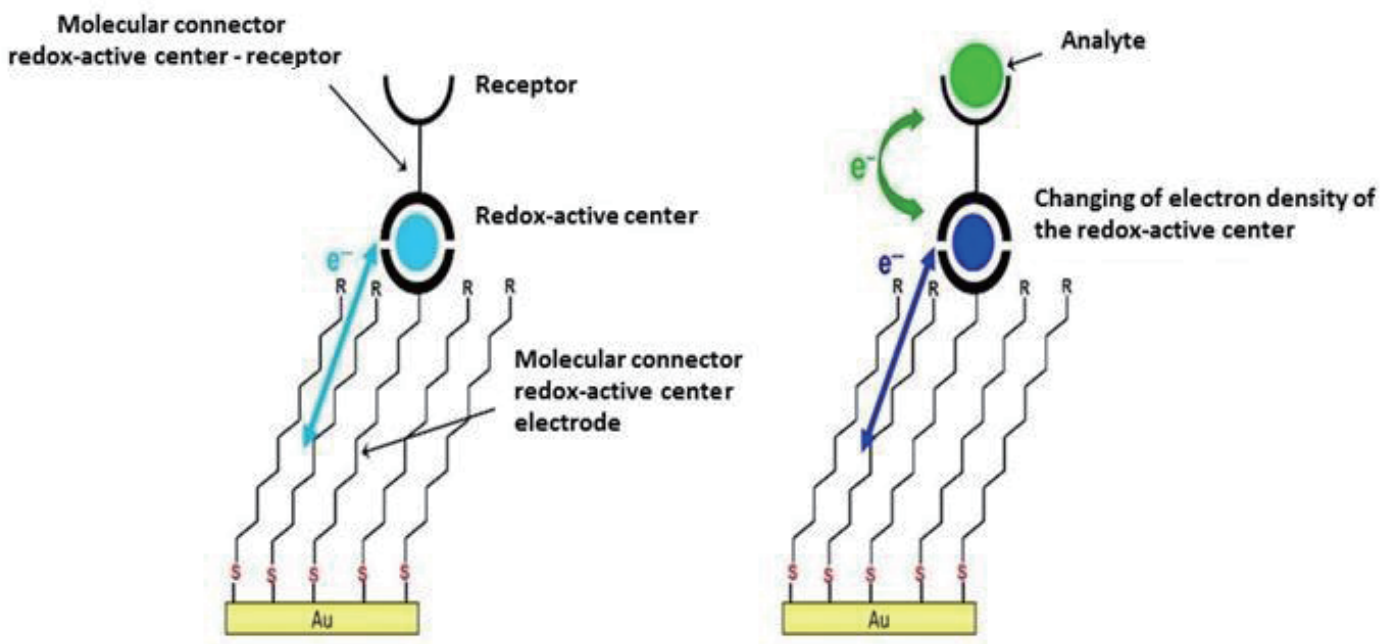

Fig. 1. (Color online) Schematic of electrochemical system based on redox-active monolayer for receptor-analyte recognition at aqueous/solid interphase. 
chemistry is the simplicity of the formation of homogeneous monolayers with a relatively strong bond between the immobilized molecule and the electrode surface. This approach is very useful in the fabrication of redox-active monolayers.

The very interesting aspect of the above research area concerns sensors based on bifunctional receptor molecules that contain not only binding sites, but also redox-active centers whose electron transfer reaction is coupled to the receptor complexation. Such systems are suitable for exploring host-guest molecular recognition processes occurring at solid/liquid interphases by electrochemical methods.

Electronic coupling, a key parameter that determines the rate of electron transfer reaction in the sensing system based on bi-functional receptor molecules is mainly realized through one or a combination of the following pathways: ${ }^{(7,8)}$

- space electrostatic interaction between the redox center(s) and the complexed guest molecule,

- bond communication provided typically by a conjugated chemical bond linkage between the redox center(s) and the binding cavity,

- additional direct coordination bond formation between the redox center and the complexed guest molecule,

- conformationally, induced perturbation of the redox center(s) caused by the complexation of a guest molecule, and

- interference by the guest species in communication between two redox-active centers.

The desired properties of a sensing system could be acquired by the selection of the optimal length of the molecular connector between redox centers and receptors as well as between redox centers and electrode surfaces. The selection of the appropriate transition metal cation is also crucial (Fig. 1).

The redox centers responsible for analytical signal generation could be located on the outer surface of a self-assembled monolayer (SAM) or be buried in SAM. Their position has a strong effect on the mechanism of analytical signal generation and consequently on their analytical properties.

The short distance between receptors and redox centers located on the outer surface of SAM enables the efficient coupling between the complexation of the analyte and the redox processes.

The receptors designed to recognize guest molecules must couple the complexation process to the redox reaction. These two reactions must mutually affect each other. Electron insertion (reduction) or withdrawal (oxidation) from a host causes changes in redox potential. The magnitude and direction of potential changes will depend primarily on the electronic coupling mechanism and the properties of the complexed guest molecule. ${ }^{(7,9)}$

The relationship between the potential shift and the stability of the host-guest complex can be described by a thermodynamic parameter named the reaction coupling efficiency (RCE) expressed as ${ }^{(10)}$

$$
\frac{K_{1}}{K_{2}}=e^{\frac{n F \Delta E}{R T}},
$$


where $K_{1}$ and $K_{2}$ are the stability constants of the host-guest complex in different redox states, $\Delta E$ is the half-potential shift, $n$ is the number of electrons involved in redox reaction, and the other variables have their usual meanings.

This equation connects the stability constant ratio $K_{1} / K_{2}$, i.e., RCE, with experimentally measurable potential shifts. Thus, it can be concluded that RCE provides information about the efficiency of interphase "host-guest" binding.

For cation receptors, the complexation reaction leads to positive potential shifts. Therefore, $\mathrm{RCE}$ is smaller than unit. The anion complexations are associated with negative potential shifts, resulting in RCE values larger than unit.

Equation (1), after the transformation reported by Gobi and Ohsaka, enables us to calculate the interface binding constants: $:^{(11)}$

$$
\exp \left\{\Delta E^{\circ}(-n F / R T)\right\}-1=K_{1}[X]+K_{1} K_{2}[X]^{2},
$$

where $R, F$, and $T(293.15 \mathrm{~K})$ have their usual meanings, $n$ is the number of electrons transferred, and $[X]$ represents the concentration of the target compound.

The formal potential $E^{\circ \prime}$ was estimated as the average of anodic and cathodic peak potentials. $\Delta E^{\circ \prime}$ represents the difference in formal potential in the absence and presence of the target compound. When $\exp \left\{\Delta E^{\circ}(-n F / R T)\right\}-1$ increased linearly with the concentration of the target compound, $K_{1}$ could be estimated and indicated 1:1 receptor:target compound complex formation. A bi-exponential plot of $\exp \left\{\Delta E^{\circ \prime}(-n F / R T)\right\}-1$ vs target compound concentration allows us to estimate $K_{1}$ and $K_{2}$. In such cases, the formation of 1:1 and 1:2 receptor:target compound complexes could be assumed.

\section{Ultrasensitive Electrochemical Anion Sensing in Water Based on Redox-active Platforms}

The geometrical diversity, sensitivity to $\mathrm{pH}$, and low charge-to-radius ratio make designing anion receptors more challenging in comparison with cation receptors. The water molecules can act as both hydrogen bond donors and acceptors, causing less anion binding in aqueous media.

There are many examples of anion receptors based on ammonia, squaramide, cryptands, and guanidinium groups. ${ }^{(12)}$ However, the anion binding of most of them occurs only in organic solvents.

From the points of view of medical diagnosis and environmental protection, it is especially important to develop sensitive and selective systems working in aqueous solutions. The intensive development of electrochemical sensors for anion detection in aqueous media was reported in the review article by Hein et al. ${ }^{(13)}$

We proposed chloride anion recognition using a dipyrromethene (DPM)-modified dipodal anion receptor attached to an electrochemically active DPM-Cu(II) or DPM-Co(II) complex (Fig. 2). Both sensing systems were able to detect chloride anions in a highly diluted water medium (in the picomolar range). ${ }^{(14)}$ 


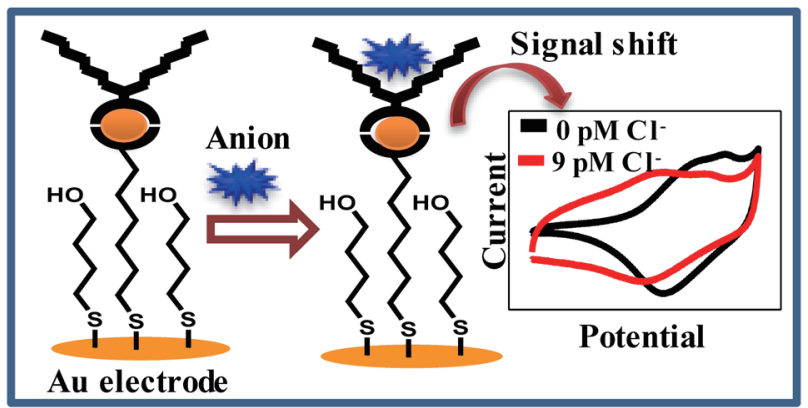

Fig. 2. (Color online) Schematic of gold electrode modified with mixed SAM incorporating mercaptobutanol and the redox-active complex DPM- $M-$ DPM dipodal anion receptor and its electrochemical response toward chloride. $M$ : $\mathrm{Co}^{2+}$ or $\mathrm{Cu}^{2+}$ (with permission from American Chemical Society).

The decrease in redox current and the shift in redox peak potential after the complexation of anions by the anion receptor are consequences of the changes in the accessibility of counterions from the supporting electrolyte to the redox center, in electron transfer efficiency to/from the underlying electrode, and in electrostatic interaction through space between the central metal and target anion in the complex. . $^{(70)}$

Gold electrodes modified with a unique cyclopeptide effective for anion recognition via the creation of hydrogen bonds have been used for sulphate sensing (Fig. 3). ${ }^{(15)}$

The cyclopeptide was connected to the surface of a gold electrode via a DPM-Cu(II) or DPM-Co(II) complex. The presence of the target anion caused the redox current decrease assisted by potential shifts for both types of sensor, enabling the sensitive detection of $\mathrm{SO}_{4}{ }^{2-}$ in the $\mathrm{pM}$ range and the linear response between 2 and $10 \mathrm{pM}$.

$\mathrm{The} \mathrm{Cu}^{2+} / \mathrm{Cu}^{+}$redox process changes the charge of the monolayer from neutral to negative, which should be compensated for by cations from the supporting electrolyte. The surface charge of the electrode containing $\mathrm{Co}^{2+}$ changes from neutral to positive when $\mathrm{Co}^{2+}$ is oxidized to $\mathrm{Co}^{3+}$. In this case, anions from the supporting electrolyte are involved in charge compensation. The proposed mechanism of electrochemical signal generation is illustrated in Fig. 4.

The cyclopeptide functionalized with a disufide group was applied in the design of a sensor working in the "ion channel mimetic" mode. ${ }^{(16-18)}$ In this system, the presence of a redox marker in the sample solution is necessary. The formation of a receptor-anion complex having a negative charge suppresses the migration of a negatively charged redox marker toward the electrode surface. This phenomenon is the basis of analytical signal generation. The presented sensor exhibits a linear dynamic range for the sulphate present in aqueous media from 0.5 to $2.0 \mathrm{pM} .^{(15)}$ The detection limit of $0.25 \mathrm{pM}$ is very similar to the value obtained for sulphate sensors based on redox-active monolayers. ${ }^{(15)}$

The sensing platform incorporating DPM-Co(II)-DPM centers poses a higher selectivity than the platform incorporating DPM-Cu(II)-DPM. This phenomenon was observed for chloride $^{(14)}$ and phosphate ${ }^{(15)}$ sensors.

The redox-active platforms have universal properties. They are suitable for the immobilization of any receptors responsible for selective analyte sensing. The redox centers are 


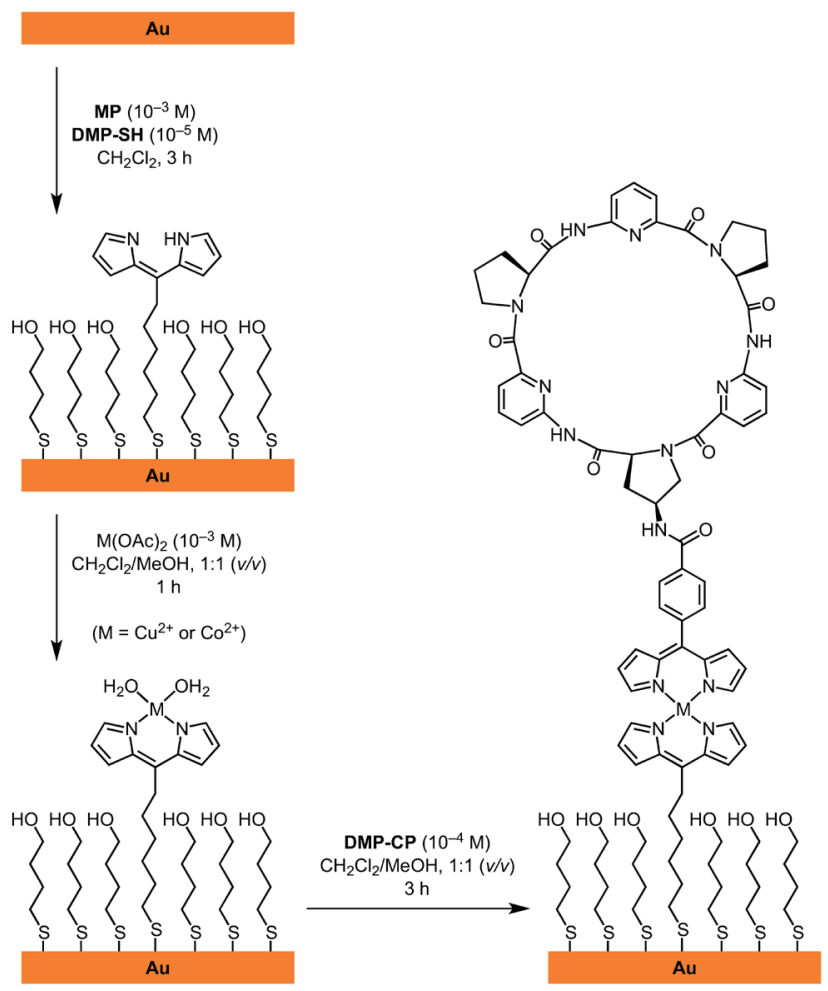

Fig. 3. (Color online) Steps of gold electrode modification with mixed SAM incorporating mercaptobutanol and redox-active complex DPM- $M-\mathrm{DPM}$ cyclopeptide anion receptor $\left(M=\mathrm{Cu}^{2+}\right.$ or $\left.\mathrm{Co}^{2+}\right)$ (with permission from Elsevier).

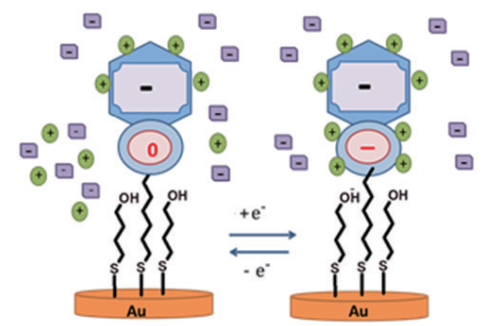

\section{Cations from the supporting electrolyte are involved}

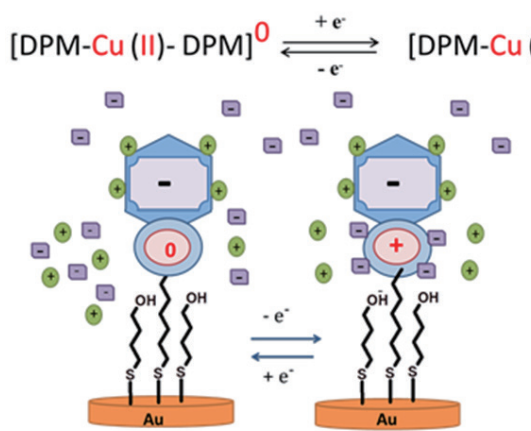

Anions from the supporting electrolyte are involved

[DPM-Co (II)-DPM] $^{0} \stackrel{-e^{-}}{\rightleftarrows}[\text { [DPM-Co (III)- DPM }]^{+}$

Fig. 4. (Color online) Schematic of the electrochemical signal generation mechanism of the designed electrodes (with permission from Elsevier). 
responsible for generating the analytical signal. Thus, there is no need to include redox markers in the sample solutions.

The selectivity sequence of the presented anion sensors correlates well with the receptor-anion binding affinity and RCE.

The explanation for such unexpected sensitivity and very high binding constants is the fact that anion recognition takes place at the aqueous/modified electrode interphase. The interphase bindings did not require full anion dehydration. This strongly facilitates their complexation by the receptors attached to the electrodes. ${ }^{(14-18)}$

\section{Electrochemical Genosensors}

\subsection{Genosensors based on redox-active monolayers}

In the majority of the recently developed electrochemical DNA or PNA sensors, the redox centers are located at the end of an ssDNA strand directed toward the solution phase. In such a situation, the hybridization process alters the distance between the redox label and the electrode surface. ${ }^{(19)}$

We proposed the DNA sensing system based on the redox-active monolayers incorporating DPM-Cu(II)-DPM-COOH and DPM-Co(II)-DPM-COOH suitable for the amide coupling of the 20-mer DNA probe functionalized with a $\mathrm{NH}_{2}$ group. ${ }^{(20)}$ In the proposed system, the redox centers are bound to the electrode surface; thus, their distance from the electrode surface remains constant. In this case, the proposed sensors generate an analytical signal because changes in the environment around the redox centers occur as a result of hybridization processes. This novel mechanism of electrochemical signal generation was named "ionbarrier switch off". The proposed genosensors have been tested for the determination of oligonucleotide sequences specific for the avian influenza virus (AIV) H5N1 with a detection limit in the $\mathrm{pM}$ range. The sensing system based on DPM-cation-DPM-COOH SAMs has universality and may be used for the amide coupling of any $\mathrm{NH}_{2}$-ssDNA probes when designing genosensors for medical diagnosis.

Joining the intensive research on the development of a universal, simple, and efficient method of forming a redox-active layer on a gold electrode surface, we applied the spontaneous reaction between the amine and epoxy groups. This type of reaction belongs to the larger family of "click" chemistry and is called the nucleophilic opening of the oxirane ring. ${ }^{(21)}$

The main advantages of click chemistry are good yield, the availability of a wide range of starting materials, and that the process is carried out under mild reaction conditions, independent of the presence of oxygen and water. Another sensor based on the ion-barrier switch off mechanism has been developed in our laboratory using epoxy derivatives of phenanthroline. ${ }^{(22)}$

The redox-active monolayer consisting of a (phenanthroline) Fe(III) complex was created on a gold electrode surface functionalized with aminoethane thiol (Fig. 5).

The $\mathrm{NH}_{2}$-ssDNA probes were attached to the surface via the epoxy group. This genosensor displays good sensitivity, with detection limits of 73 and $0.87 \mathrm{pM}$ for the 20-mer DNA and 

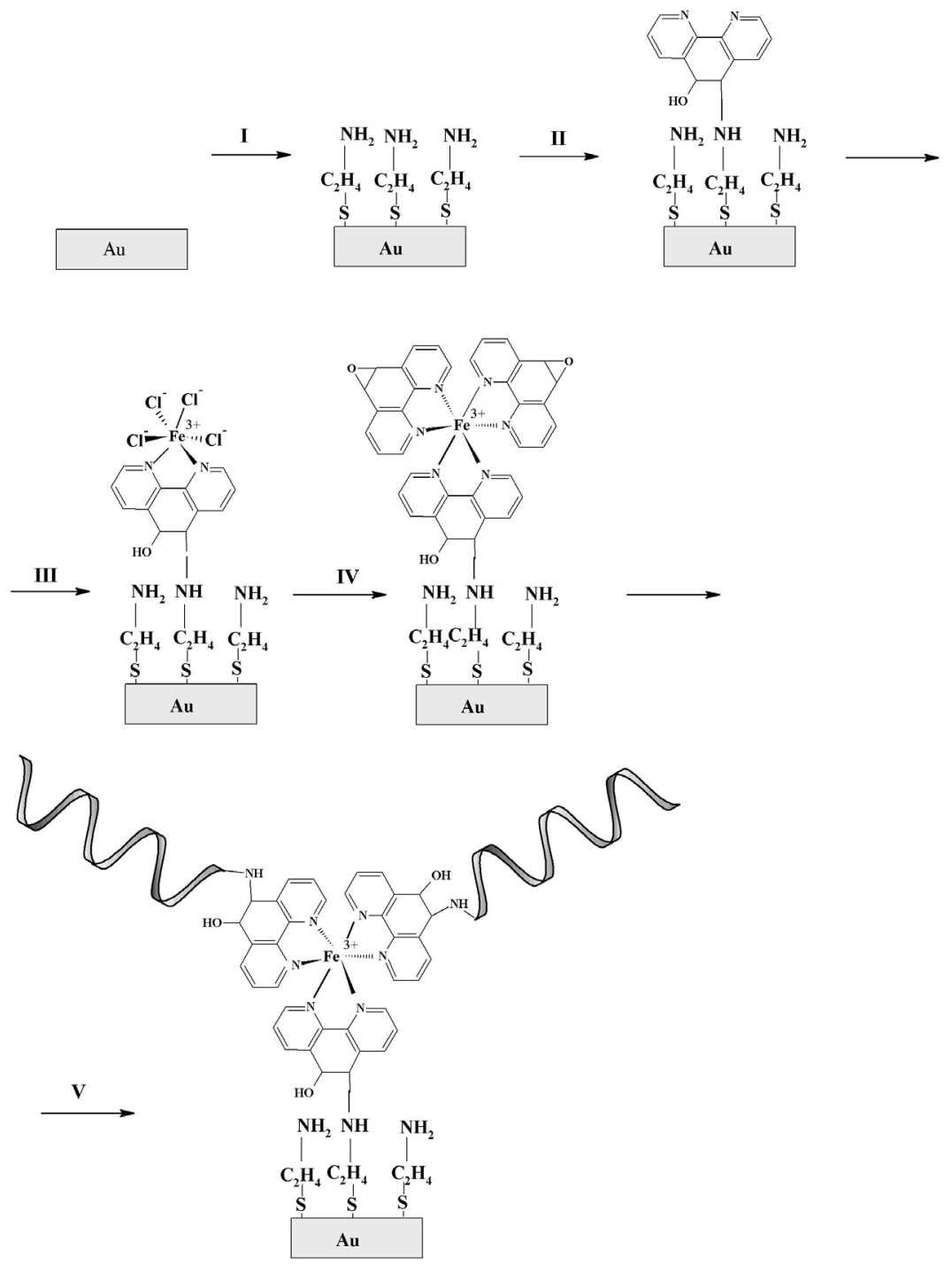

Fig. 5. Successive steps of genosensor preparation. I: AET layer formation. II: Click reaction between amine groups from AET SAM and epoxy groups from Phen-Epoxy SAM. III: Fe(III) complexation. IV: Complexation with Phen-Epoxy to form Phen-Epoxy-Fe(III)-(Phen-Epoxy) $)_{2}$ SAM. V: Click reaction between amine groups of $\mathrm{NH}_{2}-\mathrm{NC}_{3}$ (or EA) and epoxy groups of Phen-Epoxy-Fe(III)-(Phen-Epoxy) $)_{2} \mathrm{SAM}$ (with permission from Elsevier).

283-mer RNA sequences, respectively. It is worth to underline that the main advantage of the presented genosensor is its suitability for the determination of RNA sequences in biological samples, without the need of viral RNA to DNA sequence transcription.

\subsection{Genosensors based on single-strand DNA probe functionalized with redox-active label}

The next group of sensors based on redox-active layers consists of genosensors consisting of single strands of a DNA (ssDNA) probe labeled with a redox-active marker. Usually, the marker is located at the end of the ssDNA. 
The difference in flexibility between a single-stranded DNA and a double-stranded DNA is the basis of signal generation by this type of genosensor. Before hybridization, the redox marker is located very close to the electrode surface owing to the flexibility of the singlestranded DNA. It facilitates the exchange of electrons between the redox center and the electrode surface. ${ }^{(23,24)}$ After hybridization, double-stranded DNA (dsDNA) strands with rigid structures are formed. As a consequence, the distance from the marker to the electrode surface changes. This hinders the progress of the redox reaction, resulting in the switching "off" of the electrochemical signal (decrease in current). Generally, the sensors generating analytical signals according to the described mechanism are called E-DNA sensors, which are very sensitive and selective. They attained detection limits in the range of $10 \mathrm{pM}^{(19,23,24)}$

E-genosensors based on the signal-on (increase in current) mechanism are less frequently reported. Immoos et al. described the first E-genosensor, which generated the analytical signal by the signal-on mechanism. ${ }^{(25)}$ Their sensor consisted of two strands of DNA. One of them is the capture strand and the other is the probe strand decorated with ferrocene ( $\mathrm{Fc})$. The two strands are linked together by a flexible linker. Both strands are complementary towards the target DNA. As a consequence of probe hybridization with the target DNA, the labeled end of the probe is moved close to the electrode surface. Such a change facilitates electron exchange between the redox marker and the electrode surface. This sensor achieved a detection limit of $200 \mathrm{pM}$.

In our laboratory, we developed a genosensor simultaneously using the signal-off and signalon mechanisms (Fig. 6). ${ }^{(26)}$

Two different sequences of ssDNA, one decorated with $\mathrm{Fc}$ and the other decorated with methylene blue (MB), were used for gold electrode modification via $\mathrm{S}-\mathrm{Au}$ bonds. Both strains were immobilized covalently on the electrode surface.

SH-ssDNA-Fc probes create the dimers, and because of this, the redox marker is located far away from the electrode surface. After hybridization with complementary sequences, the dimer is disassembled, and one very elastic ssDNA strain transfers the redox marker very close to the electrode surface, causing a current increase in accordance with the signal-on mode.
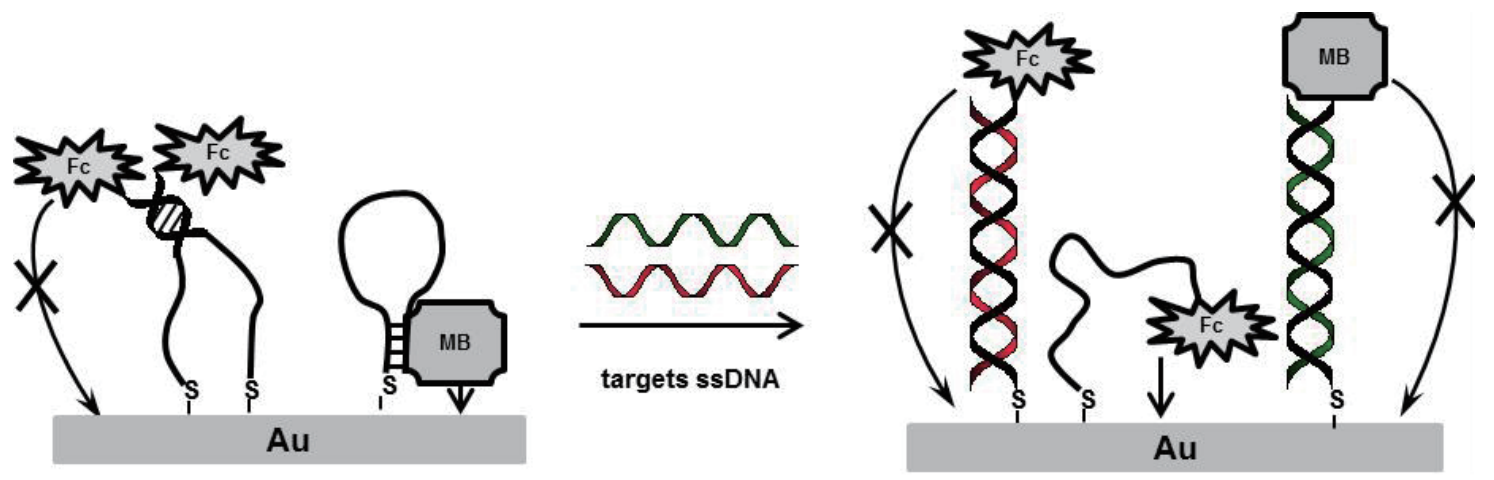

Fig. 6. (Color online) Schematic of working principle of signal-on and signal-off modes of genosensor consisting of two different ssDNA probes decorated with Fc and MB (with permission from American Chemical Society). 
The formation of the above-mentioned dimers (ssDNA-MB-ssDNA-MB) is less probable. Therefore, after the hybridization and formation of rigid dsDNA, MB is transferred far away from the electrode surface, causing current suppression. Therefore, the signal is generated in accordance with the signal-off mechanism. The duo-genosensor was selective with similar limits of detection (18-21 $\mathrm{nM}$ range) for both oligonucleotide sequences derived from genes encoding the hemagglutinin and neuraminidase of the AIV H5N1. ${ }^{(26)}$

The next group of genosensors is based on the ssDNA probe functionalized with redox markers located very close to the electrode surface. Thus, the change in their distance to the electrode surface after hybridization is rather limited.

The gold electrodes modified with the oligonucleotide probe functionalized with 3-iron bis(dicarbollide) or cobalt(II) porphyrin complex belong to this type of genosensor. ${ }^{(27,28)}$

The formation of the double helix on the electrode surface caused changes in the thickness of the double layer at the interface between the electrode surface and the sample solution. As a consequence, the 3-iron bis(dicarbollide) or cobalt(II) porphyrin complex, which is attached to the bottom of the Foligonucleotide probe, becomes more immersed in the doublehelix microenvironment. Moreover, the molecular environment around the redox-active center becomes more polar owing to the increase in negative charge at the interface. The accessibility of anions present in the supporting solution by the redox centers for balancing the charge is restricted. Therefore, electron transfer was hindered. A similar phenomenon was reported by Khor et al. ${ }^{(29)}$ The restriction of access to the counterions by the redox probe owing to the association or dissociation of the antibody at the sensing interface changed the Fc electrochemistry.

Both genosensors displayed good selectivity and sensitivity of the $\mathrm{fM}$ range to complementary targets. ${ }^{(27,28)}$ The incorporation of gold nanoparticles (AuNPs) into the sensing platforms increases their surface area. As a consequence, a higher number of receptors could be immobilized. In order to increase the number of ssDNA probes immobilized on the sensing surface and improve the hybridization efficiency, AuNPs combined with cobalt(II)-porphyrinlabelled ssDNA probes have been incorporated to fabricate genosensors (Fig. 7). ${ }^{(30)}$ With this sensing system, a detection limit of $3.8 \mathrm{aM}$, ca. 1000 times higher than that of a genosensor without AuNPs, ${ }^{(28)}$ was obtained.

\section{Redox-active Platforms for Exploring Protein-Ligand Interactions}

Properly immobilizing protein receptors on the sensing surfaces, keeping their correct orientation and physiological activity, is the main problem for biosensor design. ${ }^{(31)}$ Many proteins produced by biotechnological methods possess a histidine (His) tag. In 2013, we discovered that a gold electrode modified with an electroactive monolayer containing a thiol derivative of iminodiacetic acid (IDA) complex with $\mathrm{Cu}$ (II) was suitable for the oriented and stable immobilization of $\mathrm{rHis}_{6}$-Riol through covalent bonds formed between $\mathrm{Cu}$ (II) centers and imidazole nitrogen atoms of the His tag. ${ }^{(32)}$

$\mathrm{The} \mathrm{Cu}(\mathrm{II})$ centers deposited on the electrode surface play double roles: immobilization sites for His-tagged proteins and transduction centers to track protein-small molecule interactions. 

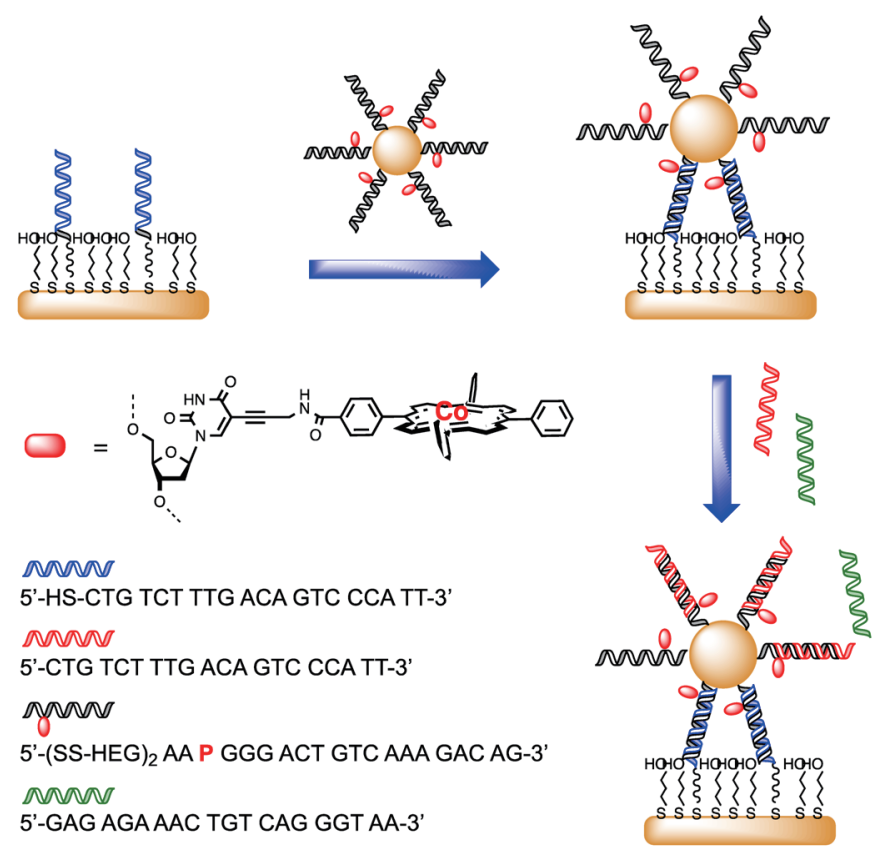

Fig. 7. (Color online) Schematic of genosensor fabrication. The capture strand (blue) is attached via a single thiol modification; the probe strand (black) containing porphyrin $(\mathrm{P})$ is anchored by two repeating disulfide and hexaethylene glycol spacer units (with permission from Royal Society of Chemistry).

The interactions between HHis $_{6}$-Riol attached to the electrode surface via an IDA- $\mathrm{Cu}$ (II) complex and kinase inhibitors present in the aqueous solution were monitored by Osteryoung square wave voltammetry. The basis of the biosensor response was the changes in the electrochemical properties of IDA-Cu(II) redox centers upon the formation of the $\mathrm{rHis}_{6}-\mathrm{Riol}_{-}$ inhibitor complex.

This new analytical system, because of its simplicity and very low compound consumption, promoted the development of numerous biosensors destined for the exploration of interphase interactions between His-tagged protein receptors attached to the sensor surface and specific ligands present in aqueous solutions. These types of biosensor were used for screening kinase inhibitors $\mathrm{JAK}^{(33)}$ and determining the concentrations of different forms of peptides (A $\beta_{1-40}$ and $\mathrm{A} \beta_{1-42)},{ }^{(34)}$ protein $\mathrm{S} 100 \beta,{ }^{(35)}$ and glycated albumin. ${ }^{(36)}$

The DPM-Cu(II) complex together with $\mathrm{His}_{6}$-H5HA antigen was also successfully applied to design an immunosensor for the determination of antibodies against AIVH5N1 (Fig. 8). ${ }^{(37)}$ This biosensor was able to detect a humoral response in the sera of hens immunized with DNA vaccine on the basis of the sequence of HA from the H5N1 virus. Its sensitivity was about 20 to 200 times higher than that of ELISA. Therefore, hens vaccinated against the AI virus could be distinguished from nonvaccinated ones by using this sensing system. Consequently, it will be very effective in the detection of antibodies for immunity surveillance and the monitoring of the efficiency of poultry vaccination programs.

The above-mentioned examples of biosensors proved the suitability of using transition metal complexes deposited on gold electrode surfaces for the stable immobilization of His- 


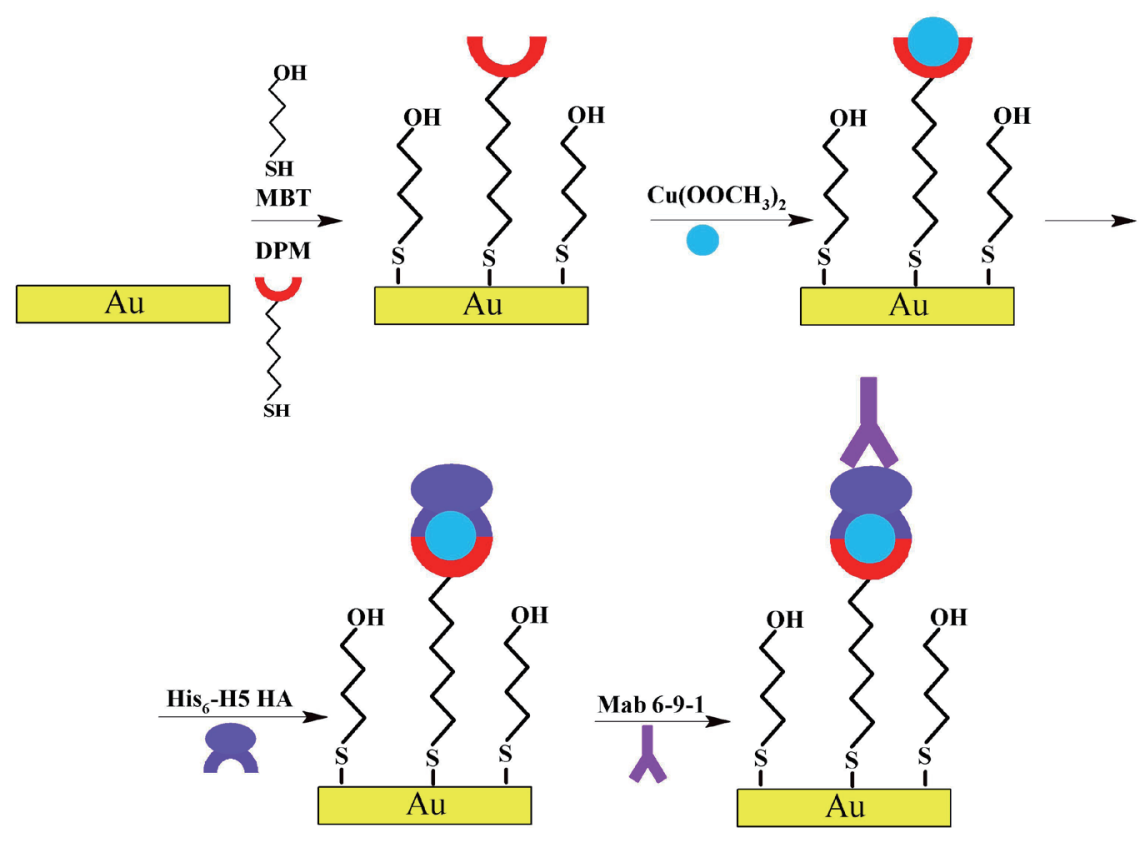

Fig. 8. (Color online) Steps of biosensor formation (with permission from Springer).

tagged protein receptors on the sensing platforms while preserving their correct orientation and physiological activity. Such analytical systems could be used for simple and efficient environmental control and medical analysis.

\section{Conclusions}

The electrochemical sensors based on redox-active layers are relatively new sensing devices. One of their main advantages is the lack of the need to use an external redox marker. The application of redox centers in such sensors creates highly interesting possibilities. These centers can simultaneously act as binding sites for host molecule immobilization and as transducers responsible for monitoring host-guest interactions.

The presented sensors showed very good sensitivity in the pM range. The explanation for such unexpected values is the fact that analyte recognition takes place at the aqueous/modified electrode interphase. The interphase binding did not require full analyte dehydration. This greatly facilitates their complexation by the receptors attached to the electrodes.

Because the examined electrochemical sensors had very good sensitivity (in the pM range), very low sample consumption (at the $\mu$ level), no effect of the matrix, simple operation, and reasonable cost, the sensors are promising analytical tools suitable for medical diagnosis and environmental and food quality control.

\section{Acknowledgments}

This work was supported by National Science Centre, Poland, No 2016/21/B/ST4/03834. 


\section{References}

1 J. M. Lehn: Supramolecular Chemistry: Concepts and Perspectives (Wiley-VCH, Weinheim, 1995).

2 V. Vogiazi, A. de la Cruz, S. Mishra, V. Shanov, W. R. Heineman, and D. D. Dionysio: ACS Sens. 4-5 (2019) 1151. https://doi.org/10.1021/acssensors.9b00376

3 A. L. Eckermann, D. J. Feld, J. A. Shaw, and T. J. Meade: Coord. Chem. Rev. 254 (2010) 1769. https://doi. org/10.1016/j.ccr.2009.12.023

4 H. O. Finklea: In Electroanalytical Chemistry, Eds. A. J. Bard and I. Rubinstein (Marcel Dekker Inc., New York, 1996) Vol. 19.

5 P. E. Laibinis, G. M. Whitesides, D. L. Allara, Y. T. Tao, A. N. Parikh, and R. G. Nuzzo: J. Am. Chem. Soc. 113 (1991) 7152. https://doi.org/10.1021/ja00019a011

6 D. L. Allara: Biosens. Bioelectron. 10 (1995) 771. https://doi.org/10.1016/0956-5663(95)99215-7

7 P. D. Beer, P. A. Gale, and Z. Chen: Adv. Phys. Org. Chem. 31 (1999) 1. https://doi.org/10.1016/S0065$3160(08) 60192-6$

8 P. A. Gale, N. Busschaert, C. J. E. Haynes, L. E. Karagiannidis, and I. L. Kirby: Chem. Soc. Rev. 43 (2014) 205. https://doi.org/:10.1039/C3CS60316D

9 P. D. Beer and P. A. Gale: Angew. Chem. Int. Ed. 40 (2001) 486. https://doi.org/10.1002/15213773(20010202)40:3<486:AID-ANIE486>3.3.CO;2-G

10 P. D. Beer, P. A. Gale, and G. Z. Chen: Coord. Chem. Rev. 185 (1999) 3. https://doi.org//10.1016/S00108545(98)00246-X

11 K. V. Gobi and T. Ohsaka: J. Electroanal. Chem. 485 (2000) 61. https://doi.org/10.1016/S0022-0728(00)00101-7

12 S. Kubik, R. Kirchner, D. Nolting, and J. Seidel: J. Am. Chem. Soc. 124 (2002) 12752. https://doi.org/10.1021/ ja026996q

13 R. Hein, P. D. Beer, and J. J. Davis: Chem. Rev. 120 (2020) 1888. https://doi.org/10.1021/acs.chemrev.9b00624

14 B. Kaur, C. A. Erdmann, M. Daniëls, W. Dehaen, Z. Rafiński, H. Radecka, and J. Radecki: Anal. Chem. 89 (2017) 12756. https://doi.org/10.1021/acs.analchem.7b03001

15 P. Gołębiewski, B. Puciłowski, F. Sommer, S. Kubik, M. Daniels, W. Dehaen, U. Sivasankaran, K. G. Kumar, H. Radecka, and J. Radecki: Sens. Actuators, B 285 (2019) 536. https://doi.org/10.1016/j.snb.2019.01.083

16 P. Gołębiewski, F. Sommer, S. Kubik, H. Radecka, and J. Radecki: J. Electroana.l Chem. 812 (2018) 249. https://doi.org/10.1016/j.jelechem.2018.01.035

17 K. P. Xiao, P. Bühlmann, and Y. Umezawa: Anal. Chem. 716 (1999) 1183. https://doi.org/10.1021/ac9809635

18 Y. Umezawa and H. Aoki: Anal. Chem. 76 (2004) 320A. https://doi.org/10.1021/ac0416259

19 H. Radecka and J. Radecki: in DNA in Supramolecular Chemistry and Nanotechnology. Eds. E. Stulz and G.H. Clever (John Wiley \& Sons, 2015) Chap.3.1, pp 140-157.

20 K. Kurzątkowska, A. Sirko, W. Zagórski-Ostoja, W. Dehaen, H. Radecka, and J. Radecki: Anal. Chem. 87 (2015) 9702. https://doi.org/10.1021/acs.analchem.5b01988

21 H. C. Kolb, M. G. Finn, and K. B. Sharpless: Angew. Che. Intel. Ed. 40 (2001) 2004. https://doi. org/10.1002/1521-3773(20010601)40

22 K. Malecka, A. Stachyra, A. Góra-Sochacka, A. Sirko, W. Zagórski-Ostoja, W. Dehaen, H. Radecka, and J.Radecki: Biosens. Bioelectron. 65 (2015) 427. https://doi.org/10.1016/j.bios.2014.10.069.

23 F. Ricci, R. Y. Lai, and K. W. Plaxco: Chem. Comm. (2007) 3768. https://doi.org/10.1039/b708882e

24 A. A. Lubin, R. Y. Lai, B. R. Baker, A. J. Heeger, and K. W. Plaxco: Anal. Chem. 78 (2006) 5671. https://doi. org/10.1021/acs.analchem.6b02376

25 C. E. Immoos, S. J. Lee, and M. W. Grinstaff: J. Am. Chem Soc. 126 (2004) 10814. https://doi.org/10.1021/acs. analchem. $8 \mathrm{~b} 02196$

26 I. Grabowska, K. Malecka, A. Stachyra, A. Góra-Sochacka, A. Sirko, W. Zagórski-Ostoja, H. Radecka, and J. Radecki: Anal. Chem. 85 (2013) 10167. https://doi.org/10.1021/ac401547h

27 I. Grabowska, A. Stachyra, A. Góra, A. Sirko, B. Olejniczak, Z.J Leśnikowski, J. Radecki, and H. Radecka: Biosens. Bioelectron. 51 (2014)170. https://doi.org/10.1016/j.bios.2013.07.026

28 I. Grabowska, D. G. Singleton, A. Stachyra, A. Góra-Sochacka, A. Sirko, W. Zagorski-Ostoja, H. Radecka, E. Stulz, and J. Radecki: Chem. Commun. 50 (2014) 4196. https://doi.org/10.1039/C4CC00172A

29 S. M. Khor, G. Liu, C. Fairman, S. Iyengar, and J. J. Gooding: Biosens. Bioelectron. 26 (2011) 2038.https://doi. org/10.1016/j.bios.2010.08.082

30 B. Kaur, K. Malecka, D. A. Cristaldi, C. S. Chay, I. Mames, H. Radecka, J. Radecki, and E. Stulz: Chem. Comm. 54 (2018) 11108. https://doi.org/10.1039/c8cc05362f

31 Y. Huang, J. Xu, J. Liu, X. Wang, and B. Chen: Sensors 17 (2017) 2375. https://doi.org/10.3390/s17102375 
32 M. Mielecki, J. Wojtasik, M. Zborowska, K. Kurzatkowska, K. Grzelak, W. Dehaen, J. Radecki, and H. Radecka: Electrochim. Acta. 96 (2013) 147. http://doi.org/10.1016/j.electacta.2013.02.085

33 J. Wojtasik, M. Mielecki, K. Kurzątkowska, K. Grzelak, P. Verwilst, W. Dehaen, J. Radecki, and H. Radecka: Sens. Actuators, B 196 (2014) 223. http://doi.org/10.1016/j.snb.2014.01.095

34 A. Jargiło, I. Grabowska, H. Radecka, M. Sulima, I. Marszałek, A. Wysłouch-Cieszyńska, W. Dehaen, and J. Radecki: Electroanalysis. 25 (2013) 1185. https://doi.org/10.1002/elan.201200537

35 E. Mikuła , A. Wysłouch-Cieszyńska , L. Zhukova , M. Puchalska, P. Verwilst, W. Dehaen, J. Radecki, and H. Radecka: Sensors 14 (2014) 10650. https://doi.org/10.3390/s140610650

36 E. Mikuła, A. Wysłouch-Cieszyńska, L. Zhukova, P. Verwilst, W. Dehaen, J. Radecki, and H. Radecka: Current Alzheimer Research. 14 (2017) 345. https://doi.org/10.2174/1567205013666161108110542

37 U. Jarocka, R. Sawicka, A. Stachyra, A. Góra-Sochacka, A. Sirko, W. Zagórski-Ostoja, V. Sączyńska, A. Porębska, W. Dehaen, J. Radecki,and H. Radecka: Anal. Bioanal. Chem. 407 (2015) 7807. https://doi. org/10.1007/s00216-015-8949-y

\section{About the Authors}

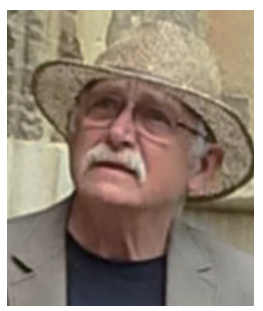

Jerzy Radecki obtained his M.Sc. degree at the Department of Organic Chemistry of Nicholas Copernicus University in Toruń in 1973. In 1980, he received a Ph.D. degree from the same university. From 1980 to 1998, he worked at the Olsztyn University of Agriculture and Technology. In 1993, he received a D.Sc. degree at this university. In 1985, he visited St. John's College of Oxford University. He spent one year (June 1989 to May 1990) as a post-doctoral fellow at the Department of Analytical Chemistry of the University of Sao Paulo. In 1990, he was granted a fellowship in the National Institute for Environmental Studies in Tsukuba, Japan. In 1993, he received the title of professor of analytical chemistry. Since 1998, he has been working as the head of the Department of Biosensors in the Institute of Animal Reproduction and Food Research of Polish Academy of Sciences in Olsztyn.

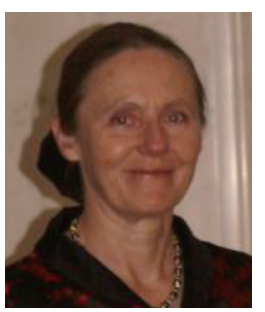

Hanna Radecka graduated from the Department of Chemistry of Nicolaus Copernicus University in Torun 1978. Since that year, she worked as an academic teacher in the Chemistry Department of the Olsztyn University of Agriculture and Technology. She received a Ph.D. degree from this university in 1986. She was a visiting scientist at Hokkaido University in Sapporo, Japan, in 1987-1988 and at The University of Tokyo in 1996, 1999, and 2001. In 2002, she spent three months at Interuniversitair Micro-Elektronica Centrum (IMEC) in Leuven (Belgium). In 1998, she joined the Department of Biosensors of the Institute of Animal Reproduction and Food Research of Polish Academy of Sciences in Olsztyn. In 2001, she successfully completed habilitation at the University of Nicholas Copernicus in Torun and became an assistant professor of analytical chemistry. In 2011, she received the title of professor of analytical chemistry and was nominated as the head of the Laboratory of Bioelectroanalysis in the Institute of Animal Reproduction and Food Research. 